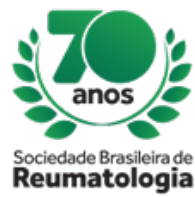

\title{
EFFECT OF ATORVASTATIN IN ENDOTHELIAL FUNCTION AND ARTERIAL STIFFNESS OF THE PATIENTS WITH SYSTEMIC AUTOIMMUNE MYOPATHIES: A RANDOMIZED, DOUBLE-BLIND, PLACEBO- CONTROLLED PILOT STUDY
}

Isabela Bruna Pires Borges (Faculdade de Medicina FMUSP, Universidade de Sao Paulo, São Paulo, SP, Brasil), Rafael Giovani Misse (Faculdade de Medicina FMUSP, Universidade de Sao Paulo, São Paulo, SP, Brasil), Diego Sales De Oliveira (Faculdade de Medicina FMUSP, Universidade de Sao Paulo, São Paulo, SP, Brasil), Alexandre Moura Dos Santos (Faculdade de Medicina FMUSP, Universidade de Sao Paulo, São Paulo, SP, Brasil), Valéria Aparecida Costa Hong (Instituto do Coração, Hospital das Clínicas, Faculdade de Medicina, Universidade de Sao Paulo, São Paulo, SP, Brasil), Luis Aparecido Bortolotto (Instituto do Coração, Hospital das Clínicas, Faculdade de Medicina, Universidade de Sao Paulo, São Paulo, SP, Brasil),

Samuel Katsuyuki Shinjo (Faculdade de Medicina FMUSP, Universidade de Sao Paulo, São Paulo, SP, Brasil)

\section{BACKGROUND}

High frequency of cardiovascular diseases and theirs risk factors, including dyslipidemia, have been described in systemic autoimmune myopathies (SAMs). These comorbidities can lead to endothelial dysfunction and arterial stiffness. Of note, statins have been shown to improve endothelial function as well as reduce arterial stiffness. Nevertheless, statins effect in patients with SAMs has never been studied. To assess the impact of atorvastatin on the endothelial function and vascular stiffness of the patients with SAMs.

\section{MATERIALS AND METHODS}

This prospective, double-blind, randomized, placebo-controlled pilot study included 21 consecutive adult pre-menopaused female patients with SAMs (EULAR/ACR 2017 and Connors et al., 2010), clinically stable and with dyslipidemia, between 2017 and 2018. Patients with other systemic autoimmune diseases (overlap syndromes), diabetes mellitus or previous lipid-lowering agent using were excluded. Patients were randomized to receive atorvastatin $20 \mathrm{mg} /$ day or placebo for 12 weeks. The status of the disease was assessed using the International Myositis Assessment \& Clinical Studies Group (IMACS) set scores. Flow-mediated vasodilation (FMD) was achieved from brachial arterial above the antecubital fossa (Doppler ultrasound), whereas carotid-femoral vessels pulse wave velocity (PWV) was performed by Complior ${ }^{\circledR}$ system. These evaluations were assessed at the baseline and after 12 weeks.

\section{RESULTS}

From 21 randomized patients, 5 patients were excluded during the study, remaining 16 patients (12 in the atorvastatin group and 4 in the placebo group): 9 with dermatomyositis and 7 with anti-synthetase syndrome. General data (demographics, disease status, comorbidities, treatment, endothelial function and properties of large vessels) at baseline were comparable between the atorvastatin group and placebo $(P>0.05)$. After 12 weeks of follow-up, there were no differences between the group treated and contrlol, including the median values of the endothelial function parameters [6.3 (1.7-8.6) vs. $1.7(0.0-3.5) \%$, $\mathrm{P}=0.177]$ and vascular stiffness [7.4 (6.7-8.1) vs. $7.9(7.0-8.2) \mathrm{m} / \mathrm{s}, \mathrm{P}=0.539]$.

\section{CONCLUSION}

In the present study, the use of atorvastatin did not promote changes in the values of the endothelial function and arterial stiffness in patients with stable SAMs. More studies are necessary to corroborate our findings.

NCT number 03092154. 
Support: Fundação de Amparo à Pesquisa do Estado de São Paulo (FAPESP) \#2016/20371-1 and \#2017/13109-1. 